\title{
Considerações sobre felicidade por Clusters de discentes de administração em uma instituição pública de ensino superior
}

Objetivou-se investigar a percepção de felicidade de discentes de uma IES. Para tanto, identificou-se a felicidade em função de variáveis sócio demográficos, agrupando-as a fim de descrever o comportamento dessas na distinção de dois grupos (mais felizes e menos felizes). Realizou-se pesquisa de campo, com suporte na abordagem quantitativa, cujos dados foram coletados em uma amostra probabilística de 201 discentes, de um universo de 319 , vinculados ao curso de Administração, com base em instrumento composto por quatro escalas referentes à felicidade. Realizou-se estatística descritiva e Análise de Cluster (AC), este com base no método K-Means e análise de variância (ANOVA) a fim de constituir grupos com variáveis significativas para a formação dos clusters. No primeiro predomina discentes mais maduros, mais distribuídos nos semestres finais do curso e que, em geral, trabalham. Nesse grupo a satisfação com a vida, se comparada com o grupo 2 é menor, porém há maior percepção de felicidade subjetiva. No segundo prepondera jovens que estão iniciando o curso superior, e que, em geral, não trabalham. São mais satisfeitos com a vida, embora menos felizes do que o grupo 1. Ressalta-se que avaliar-se em condições excelentes de vida -item 3 (Satisfação com a vida) e avaliar-se como alguém que aproveita a vida ao máximo-item 9 (felicidade subjetiva) são apreciadas de forma distinta pelos grupos. Prepondera no grupo mais maduro maior percepção de felicidade subjetiva, indicativo para estudos futuros sobre uma possível relação entre maturidade e capacidade de avaliação subjetiva de felicidade.

Palavras-chave: Felicidade; Cluster; Satisfação com a vida; Felicidade subjetiva.

\section{Considerations Happiness by Cluster administration students in a public institution of higher education}

This study aimed to investigate the perception of students of happiness of an HEl. Therefore, it identified the happiness due to sociodemographic variables grouping them in order to describe the behavior of these two groups in the distinction (more happy and less happy). He conducted field research, supporting the quantitative approach, whose data were collected from a random sample of 201 students, from a universe of 319, linked to the course of Directors, based on instrument consists of four scales of happiness. A descriptive statistics and Cluster Analysis (CA), this based on the K-Means method and analysis of variance (ANOVA) to form groups with significant variables for the formation of clusters. In the first dominant mature students, distributed over the halves of the final course and which generally work. In this group, satisfaction with life, compared with group 2 is less, but there is greater awareness of subjective happiness. In the second preponderates young people who are starting college, and generally do not work. Are more satisfied with life, though less happy than group 1 . It is noteworthy that be assessed in excellent living conditions - item 3 (Life satisfaction) and to evaluate yourself as someone who enjoys life to máximo- item 9 (subjective happiness) are assessed differently by the groups. Preponderates the more mature group greater perception of subjective happiness, indicative for future studies on a possible relationship between maturity and evaluation capacity of subjective happiness.

Keywords: Happiness; Cluster; Satisfaction with life; Subjective happiness.

\section{Topic: Recursos Humanos}

Reviewed anonymously in the process of blind peer.

Fabiana Pinto de Almeida Bizarria

Universidade da Integração Internacional da Lusofonia Afro-Brasileira, Brasil http://lattes.cnpq.br/0008637422329772

bianapsq@hotmail.com

Flávia Lorenne Sampaio Barbosa

Universidade Federal do Piauí, Brasil

http://lattes.cnpq.br/3271567015867945

flsbarbosa@hotmail.com

Soraia Germana de Sousa Rocha

Universidade Federal do Piauí, Brasil

http://lattes.cnpq.br/2311156024142909

soraiagermana@gmail.com

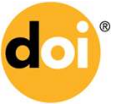

DOI: 10.6008/SPC2179-684X.2017.001.0009
Referencing this:

BIZARRIA, F. P. A.; BARBOSA, F. L. S.; ROCHA, S. G. S.. Considerações sobre felicidade por Clusters de discentes de administração em uma instituição pública de ensino superior. Revista Brasileira de Administração Científica, v.8, n.1, p.103-117, 2017. DOI http://doi.org/10.6008/SPC2179-684X.2017.001.0009 


\section{INTRODUÇÃO}

Estudos que buscam compreender a interação entre felicidade, bem-estar subjetivo e satisfação com a vida, bem como, na composição dessas variáveis com outros indicadores, estão em crescimento nas últimas décadas (COLETA et al., 2006; RODRIGUES et al., 2010; COLETA et al., 2012). 0 amparo teórico distingue duas posições sobre felicidade. Por um lado, associa o termo ao bem-estar subjetivo (DIENER et al., 2000; PASCHOAL et al., 2010), por outro é reflexo de sentimentos e cognições capazes de distinguir afetos positivos e negativos experienciados pelos indivíduos (SELIGMA, 2004).

A relevância da investigação também se confirma por ser emergente, embora situe volume expressivo de publicações (COLETA et al., 2012), sendo exemplificado por pesquisas de cunho teórico (DIENER, 2009; FERRAZ et al., 2007; PASSARELI et al., 2007; GIACOMONI, 2004; COSTA et al., 2007) e com abordagem empírica com intenções de adaptar instrumentos e dar respostas à novas hipóteses de pesquisa (ALBUQUERQUE et al., 2004; RODRIGUES et al., 2010).

Alguns achados imprimem contrariedade ao tema, como o fato da distinção de sexo não influenciar a felicidade (WILSON, 1967; GUEDEA et al., 2006), em oposição à leitura de que os homens tendem a ser mais felizes (LUCAS et al., 2000; DIENER et al., 1999), ou, ainda, de que as mulheres demonstram maiores índices de felicidade (COLETA et al., 2012). Indica-se, também, uma relação negativa com a idade (WILSON, 1967; LUCAS et al., 2000; COLETA et al., 2012); em contraposição a pesquisas, encontram uma relação positiva (OTTA et al., 2004; GONÇALVES et al., 2008) e, também, relações praticamente nulas (STOCK et al., 1983; INGLEHART, 1990), também evidenciadas no que diz respeito ao estado civil (DEMO et al., 1996; LYKKEN et al., 1996), embora entre os muito felizes há maior participação de pessoas casadas (WATSON, 2000).

No quesito condições socioeconômicas, estudos em diferentes países informam uma relação positiva entre situação financeira e felicidade, com maior impacto em países com maior índice de pobreza (INGLEMAN et al., 2000; DIENER et al., 2000; CORBI et al., 2006; OLIVEIRA et al., 2009), sendo que o estudo de Coleta et al. (2012) indica a mesma relação ao estudar sujeitos segundo a renda familiar. Fato não corroborado em estudos na América Latina, haja vista que seus índices se assemelham aos encontrados na Austrália e no Japão (VEENHOVEN, 1991). Coleta et al. (2006) declara que "os indivíduos ricos são apenas ligeiramente mais felizes que os pobres, e a beleza e a saúde física parecem ter pouco a ver com a felicidade", embora Kahneman et al. (2006) indique que as pessoas com alto poder aquisitivo não demostram ser mais felizes em comparação com a população em geral.

De forma geral, os estudos apontam que a elevação de bem-estar subjetivo e felicidade estariam atreladas à características sociais, pessoais e aspectos do país em que se vive, como, por exemplo, democracia, liberdade política, segurança, e, também, religião, saúde, trabalho, autoaceitação, e possuir rede social ampla (SELIGMAN, 2004; DIENNER et al., 2000; COLETA et al., 2006; SOLOVEY et al., 2000). Este, por exemplo, situa a compreensão de que há propagação de felicidade em grupos sociais (FOWLER et al., 2008), sendo a família o principal deles (RIBEIRO, 2009). 
No âmbito educacional, estudos indicam relação entre felicidade e melhor aproveitamento acadêmico (COLETA et al., 2006), e, também, entre felicidade, maiores níveis de titulação e de renda de docentes (COLETA et al., 2007), sendo, também, mais intensamente influenciado por fatores intrínsecos aos sujeitos das pesquisas (COLETA et al., 2012).

Pelo impacto em diversos aspectos da vida, a felicidade também se releva importante no âmbito do trabalho (CARVALHO, 2010; CARVALHO et al., 2010). Além da relação entre felicidade e saúde, investigações apontam que pessoas felizes são mais adaptáveis à situações problemáticas, em função de possuírem maior autocontrole, e, também, são mais cooperativas (LYUBOMIRSKY et al., 2005). Portanto, um baixo nível de felicidade no local de trabalho pode impactar de forma negativa a saúde, o trabalho, as relações familiares, o desempenho acadêmico, ou seja, a vida como um todo (CARVALHO, 2010).

Diante do contexto apresentado, compreende-se relevante estudos que explorem a felicidade por meio de variáveis que podem caracterizar essa experiência. A problemática se situa no âmbito da educação superior, considerando que a felicidade pode propiciar maior sucesso acadêmico (COLETA et al., 2006) e, possivelmente, uma transição para o mercado de trabalho mais harmoniosa, haja vista maior capacidade de adaptação dos felizes (LYUBOMIRSKY et al., 2005), sendo considerados, também, mais eficientes (OSWALD et al., 2009). Assim, objetivou-se investigar a percepção de felicidade de discentes de uma Instituição de Ensino Superior. Para tanto, identificou-se a felicidade em função de variáveis sócio demográficos, agrupando-as, a fim de descrever o comportamento dessas na distinção de dois grupos (mais felizes e menos felizes).

A pesquisa, ainda, avança na discussão do tema por especificar a formação discente a ser estudada, haja vista que o futuro administrador lidará com a demanda de melhor equacionar aspectos relacionados à qualidade de vida com o ambiente de trabalho, seja como trabalhador-gestor, seja como trabalhadorempregado. Assim, difundir diálogo sobre o tema no âmbito da administração ancora a contribuição da pesquisa, haja vista que a premissa de que a felicidade pode ser alcançadas por meio do trabalho estimula gestores a identificar estratégias de promoção do bem-estar e da qualidade de vida no ambiente laboral (PASCHOAL et al., 2010)

\section{REVISÃO TEÓRICA}

\section{Concepções e Mensuração da Felicidade}

Indagações sobre o que significa 'felicidade' vão além da filosofia e dos valores ocidentais, abrangendo indícios de espiritualidade e inserção na sociedade, conquista de espaços, realização de desejos, melhores relações interpessoais, maior rendimento e satisfação com o trabalho, resiliência, melhor união matrimonial e uma melhor qualidade de vida (LYUBOMIRSKY et al., 2005).

Com Sócrates, felicidade antes de origem divina passou a ser definida em função de uma responsabilidade individual, difundida por Aristóteles, que agrega ao termo a ideia de virtude, adquirida pela busca da beleza, da riqueza, da saúde e do poder (FERRAZ et al., 2007). Com o lluminismo e a Revolução 
Francesa, ainda verificada nos tempos atuais, há nova conotação à felicidade, agora, sendo expressa como direito do cidadão (FERRAZ et al., 2007; MCMAHON, 2006).

A pluralidade de conceitos é sintetizada por Cloniger (2004), que difunde a ideia de felicidade atrelada à viver de forma coerente, o que permite distanciar os sentimentos de carência e compreender os aspectos causadores da harmonia e transparência existente na humanidade, por meio de autoavaliações e avaliações contextuais (VEENHOVEN, 1997). Assim, infere-se que a autoconsciência aguçada permite a pessoa monitorar-se e observar-se em ação, o que propicia a vida mais coerente. Esta posição, de certo modo, individualiza a responsabilidade sobre a felicidade.

A felicidade também é invocada pela ideia de trajetória, em oposição à alegrias momentâneas, abrangendo aspectos sociais (CORBI et al., 2006) e econômicos (RIBEIRO, 2009). A felicidade é também relativa, em função da experiência subjetiva que responde de forma diversa as situações de existência (LYUBOMIRSKY, 2001).

Investigações sobre o tema são amparadas por estudos no domínio da Psicologia Positiva, expressando, também, os limites da experiência subjetiva relacionada ao bem-estar, satisfação, otimismo entre outros (FERRAZ et al., 2007). A felicidade, nesse escopo, revela-se no âmbito das relações afetivas e nas construções cognitivas desenvolvidas ao longo da vida. Porém, ainda há intenso esforço para melhor compreender as forças que atingem a felicidade individual (SELIGMAN et al., 2000).

Veenhoven (2007) classificou o conceito em duas dimensões, considerando a avaliação externa ou interna da felicidade. No primeiro caso, concebida como predecessor, a felicidade se situa como condições ambientais de vida, definido em função do quanto o ambiente favorece uma vida feliz (avaliação e externa) e como condições pessoais para a vida, repercutindo na capacidade de enfrentamento das situações adversas e aproveitamento das condições favoráveis (avaliação interna). Como resultado, ela pode ser concebida como uma utilidade para a vida, sendo caracterizada como a análise sobre o que é esperado do indivíduo em relação ao significado da vida (avaliação externa) e uma satisfação com a vida, que indica a apreciação subjetiva da vida (avaliação interna).

No que tange à abrangência e duração da felicidade, um cruzamento entre dois domínios (domínio específico versus domínio da vida como um todo, compõe a delimitação de quatro conceitos (VEENHOVEN, 2007): a satisfação instantânea, estando no âmbito de um domínio específico (uma viagem, por exemplo); a experiência de topo, em função da experiência como um todo, intensa, porém de curta duração; a satisfação em um domínio, demanda maior duração, embora se processe em um único domínio; e a satisfação com a vida, que representa a experiência geral de felicidade e, também, de maior duração.

A década de 1960 é enfatizada como momento importante na evolução dos estudos sobre felicidade, com importante debate metodológico (CORBI et al., 2006), situando discussões em torno da melhor abordagem de pesquisa e instrumentos de medidas para se apreender o fenômeno (SCALCO, 2008). Os principais instrumentos de mensuração foram elaborados nas décadas de 1980 e 1990, especificamente a Satisfaction With Life Scale (SWLS) (DIENER et al., 1985) e a Subjective Happiness Scale (SHS) (LYUBOMIRSKY et al., 1999). 


\section{Implicações gerenciais da felicidade}

Sendo a felicidade representante de uma vida plena (VEENHOVEN, 2007), a administração se situa como componente social que visa mediar uma relação econômica (no âmbito empresarial) e social (no campo público) diante dos desafios de inovar estrategicamente, desenvolver e reconfigurar capacidades dinâmicas e compreender o contexto organizacional dando sentido à experiência subjetiva das pessoas no mundo do trabalho (VICK, 2015). Tal assertiva aproxima-se da leitura de que o esforço humano deve guiar-se pela busca da felicidade (HUME, 1980).

Nesse sentido, a ciência administrativa depara-se com a tarefa de compreender e agir em torno das trocas simbólicas dos atores na construção da realidade (VICK, 2015), posto que a felicidade, resultante dessas trocas, associam-se com o ganho de produtividade, em função de maior adaptabilidade e criatividade agregadas ao trabalho (OSWALD et al., 2009; AMABILE, 2005; GIACOMONI, 2004). Nesse caminho, compreender a subjetividade emerge como estratégia para situar desafios organizacionais em torno da globalização, do controle de custos, situando a relevância da confiança entre stakeholders, do comportamento ético e da Responsabilidade Social Corporativa (RSC) (RODRIGUES, 2004).

Diante do fato da felicidade ser também experienciada no contexto de vida-trabalho, a qualidade de vida e a satisfação no trabalho divulgam elaborações teóricas que comungam para compreender e propiciar felicidade ao ambiente de trabalho, sendo uma condição social atrelada à ganhos de competitividade por um lado (OSWALD et al., 2009; AMABILE, 2005; GIACOMONI, 2004), pelo reconhecimento das pessoas como os recursos mais importantes da organização (PASCHOAL et al., 2010) e, também, como demanda social por maior RSC (RODRIGUES, 2004).

Por meio do trabalho, o ser humano se constitui como sujeito e afirma sua subjetividade e identidade (BENDASSOLI et al., 2013). Assim, a vivência de bem-estar, definido como felicidade, "é mais elevado quanto mais frequentemente e intensas foram as emoções positivas e quanto mais elas prevalecerem sobre o afeto negativo do trabalhador" (PASCHOAL et al., 2010). Assim, a promoção de qualidade de vida contribui de forma importante nessa concepção de felicidade do trabalho (CARVALHO et al., 2010). Nesse escopo, a formação de administradores situa a discussão em torno da felicidade no trabalho, com suporte na compreensão dos discentes como futuros gestores e, com isso, mediadores de qualidade de vida e de felicidade e, também, sujeitos-capazes-portadores de felicidade.

\section{METODOLOGIA}

Diante da perspectiva de investigar a percepção de felicidade dos discentes de uma Instituição Pública de Ensino Superior localizada no estado do Piauí, realizou-se pesquisa de campo, descritiva do tipo levantamento ou survey com suporte na abordagem quantitativa (RICHARDSON, 1999; COLLIS et al., 2006; VERGARA, 2009), cujos dados foram coletados em uma amostra probabilística de 201 discentes, de um universo de trezentos e dezenove, vinculados ao curso de Administração. Os instrumentos de pesquisa utilizados: 
A Escala de Item Único e Escala da Escada (composta também por um item), desenvolvida por Cantril (1965), objetiva a análise das condições atuais (melhor ou pior) em relação à satisfação com a vida. Rodrigues et al. (2010) e Diener (1984) ressaltam que as escalas de item único não são passíveis de análise quanto à sua confiabilidade interna, embora sua utilização seja corrente, inclusive pela utilização no German Socioeconomic Panel (GEOP), que monitora diversos aspectos sociais na Alemanha;

A Satisfaction With Life Scale (SWLS), na perspectiva de avaliar a satisfação com a vida por meio de cinco itens (escala likert unidimensional de 7 pontos) (DIENER et al., 1985). A escala propõe uma análise global da satisfação, sem distinguir domínios específicos, como satisfação com a saúde ou finanças (PAVOT et al., 1993); e

A Subjective Happiness Scale (SHS), com a formação de quatro itens (escala likert unidimensional de 7 pontos) para avaliar a felicidade subjetiva (LYUBOMIRSKY et al., 1999). O bem-estar subjetivo (BES) relaciona-se à cognição e aos afetos sobre a vida, de forma que se processe avaliações sobre a existência (SELIGMAN et al., 2000). Em relação aos itens, dois descrevem a felicidade absoluta (o quanto se considera feliz) e a relativa (o quanto se sente feliz em comparação com os outros), os outros dois itens quantificam a felicidade e a infelicidade dos sujeitos respondentes (RODRIGUES et al., 2010).

A satisfação com a vida, bem como a felicidade subjetiva, ou bem-estar subjetivo são apreciados como componentes de uma experiência de felicidade a ser mensurada pela combinação de escalas apresentada no quadro 1. Além dos instrumentos citados, o Instrumento Final (quadro 1) possui uma questão com a solicitação de três palavras associadas à felicidade. Também foram definidas cinco questões sócio demográficas (sexo, idade, participação na vida econômica, semestre do curso e objetivos profissionais).

Quadro 1: Instrumentos finais da pesquisa.

\begin{tabular}{|c|c|}
\hline Instrumento & Indicadores \\
\hline Palavras & 1. Cite 3 (três) palavras que Ihes vêm imediatamente à lembrança em relação à felicidade \\
\hline \multirow{5}{*}{$\begin{array}{l}\text { Escala de } \\
\text { Satisfação com a } \\
\text { Vida }\end{array}$} & 2. Em muitos aspectos, a minha vida se aproxima dos meus ideais. \\
\hline & 3. As minhas condições de vida são excelentes \\
\hline & 4. Estou satisfeito com a minha vida \\
\hline & 5. De modo abrangente, até agora eu consegui obter aquilo que era importante na vida \\
\hline & 6. Se pudesse viver a minha vida de novo, não alteraria em quase nada \\
\hline \multirow{4}{*}{$\begin{array}{l}\text { Escala Subjetiva } \\
\text { de Felicidade }\end{array}$} & 7. Em geral me considero uma pessoa totalmente feliz \\
\hline & 8. Comparativamente com as outras pessoas como eu, considero-me uma pessoa totalmente feliz \\
\hline & $\begin{array}{l}\text { 9. Algumas pessoas são geralmente muito felizes. Elas aproveitam a vida, aconteça o que acontecer, procurando } \\
\text { obter o máximo. Em que grau essa afirmativa se aplica a você? }\end{array}$ \\
\hline & $\begin{array}{l}\text { 10. Algumas pessoas geralmente não são muito felizes. Embora não estejam deprimidas, nunca parecem ser tão } \\
\text { felizes quanto poderia ser. Em que grau essa descrição se aplica a você? }\end{array}$ \\
\hline $\begin{array}{l}\text { Escala de item } \\
\text { único }\end{array}$ & 11. Considerando todas as coisas, você está totalmente feliz nos dias atuais \\
\hline Escala da Escada & $\begin{array}{l}\text { 12. Considere a figura de uma escada. Suponha que o topo dela represente a melhor e o chão da escada a pior } \\
\text { vida pra você. Onde na escada você sente que está no presente momento? }\end{array}$ \\
\hline \multirow{5}{*}{$\begin{array}{l}\text { Dados Sócio } \\
\text { demográficos }\end{array}$} & 13. Sexo \\
\hline & 14. Idade \\
\hline & 18. Qual a sua participação na vida econômica da família? \\
\hline & 19. Semestre que está cursando? \\
\hline & 20. Objetivo profissional \\
\hline
\end{tabular}

O tratamento dos dados, inicialmente, buscou identificar e analisar os missing values (valores perdidos), com o objetivo de identificar padrões existentes nos dados perdidos que caracterize esse processo. Nessa etapa o pesquisador tomou a decisão de ignorar ou não os dados que acontecem aleatoriamente nas variáveis (HAIR JUNIOR, 2009). Nesse sentido, optou-se por desconsiderar os missing values por serem de natureza completamente aleatória. No que se refere aos outliers, não 
houve necessidade de tratamento e correção, visto que os mesmos não interferiram no padrão dos dados a serem analisados (HAIR JUNIOR, 2009).

Realizou-se estatística descritiva para identificar a felicidade em função de aspectos sócio demográficos, e, em seguida, realizou-se Análise de Cluster (AC), diante da tarefa de agrupar as variáveis referentes à felicidade e aos aspectos sócio demográfico, descrevendo o comportamento dessas variáveis na distinção dos mais felizes e menos felizes. No primeiro caso, apresentou-se a mensuração com base nas frequências das questões e dos respondentes, como forma de compreender melhor a amostra do estudo.

Em segundo lugar, realizou-se análise $\mathrm{AC}$, com amparo no método $K-M e a n s$, utilizado para gerar o agrupamento de casos com a finalidade de apreender grupos que possuem características comuns, com grande similaridade interna e dissimilaridade externa, com base no centroide do grupo de variáveis escolhidas. O método não hierárquico de agrupamento foi definido, posto que é adequado quando se conhece a priori, a quantidade de agrupamentos para análise do estudo (POHLMANN, 2009). Nesse caso, a solução bipartite foi escolhida, por contemplar diferenças entre dois perfis de felicidade (os mais felizes e os menos felizes).

Após a definição do quantitativo de clusters, realizou-se análise de variância (ANOVA), para verificar se existem diferenças significativas entre os grupos com base na significância das variáveis (POHLMANN, 2009). Após o teste ANOVA realizou-se exclusão das variáveis não significantes, obtendo-se com isso um conjunto de variáveis e clusters mais estáveis, com agrupamentos que possuem 95\% de probabilidade de estarem corretos (POHLMANN, 2009).

Com a composição final dos clusters, realizou-se cruzamento dos casos com os grupos afim de se obter informações para definição de perfil. Este foi apreciado considerando a proporcionalidade das respostas de cada grupo em comparação com o outro. Para as questões com escala de 7 pontos, sendo o número 4 aquele que não representa maior ou menor satisfação ou felicidade subjetiva, realizou-se somatório das respostas dos itens 1-3 (menos satisfeito ou felizes) e das respostas dos itens 4-7 (mais satisfeitos e felizes). Assim, foi possível descrever com base em maior proporção de respondentes o perfil e distinção dos grupos.

\section{RESULTADOS}

A amostra do estudo (201 discentes) possui como perfil o predomínio de discentes do sexo masculino 51,7\%(104), sendo 48\%(97) mulheres. São em sua maioria jovens entre 17 e 22 anos $70 \%(141)$, tendo também a participação de $23 \%(46)$ de discentes entre 23 e 28 anos e de $4 \%(8)$ entre 29 e 24 anos. Há ainda participação de sujeitos com idades acima de 35 anos, com 3\%(6) discentes. Em relação ao estado civil, os solteiros são maioria, com $84,6 \%(170)$, em comparação com os casados $13,4 \%(27)$ e os separados $2 \%(4)$. Não há discentes viúvos. 
Os respondentes, em geral, não possuem filhos, 91\% (183), posto que apenas 9\% (18) discentes são pais. Também a maioria não trabalha, 60,2\% (121). Dos que trabalham, 15,9\% (32) dos discentes trabalham mas recebem completo da família, sendo que $23,9 \%$ (48) trabalham e mantém suas despesas. Outra questão de análise do perfil indicou que os discentes objetivam com a formação graduada, em sua maioria, o concurso público $52 \%(104)$, seguido do próprio negócio $22,5 \%$ (45), da formação pós graduação $17 \%$ (34), contratação por empresa privada $6,5 \%(13)$ e negócio familiar $2 \%$ (4). Avigora-se, com isso, uma amostra composta por um público, predominantemente, masculino, jovem, sem compromissos conjugais legais e despesas familiares e, focados na consolidação das suas carreiras por meio do concurso público.

No que diz respeito à indicação de palavras relacionadas à felicidade (item 1), evidenciou-se, com base em percentual definido com auxílio do Atlas ti., por ordem decrescente de palavras aleatoriamente elencadas pelos participantes, que a família foi a palavra mais citada com 17,91\% (108 vezes), seguido do amor com 10,28\% (68 vezes) e, logo depois, pelo dinheiro com 7,96\% (48 vezes). Convém ressaltar outras palavras citadas como por exemplo, amigos, amizade, Deus, paz, realização, satisfação, saúde, sucesso e trabalho. A aglutinação dessas palavras, de um modo geral, podem compor o contexto de felicidade dos respondentes, conforme se vê na figura 1.

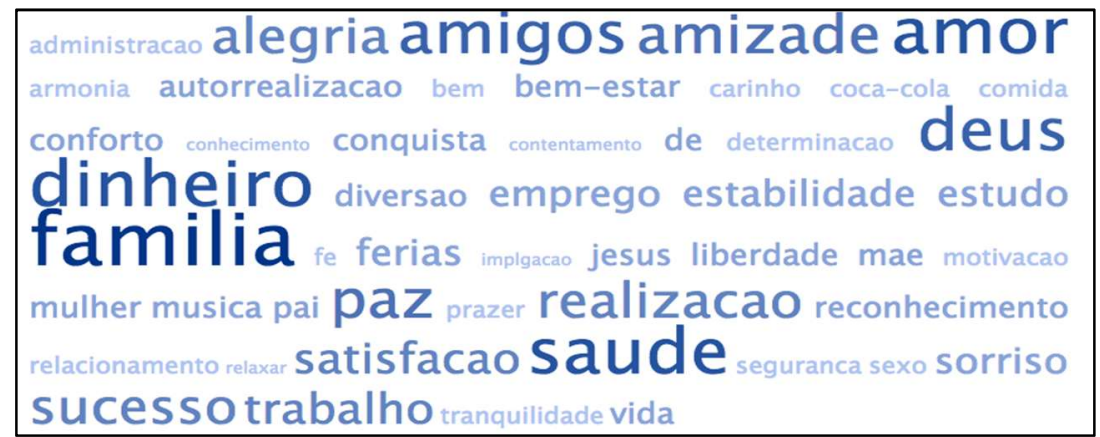

Figura 1: Disposição das principais palavras indicativas de felicidade

Para a formação dos clusters, optou-se pelo método de Análise de Cluster (AC) não hierárquico com base numa definição de solução bipartite. Os dois clusters finais formados comportam variáveis que, após a análise de variância dos grupos (ANOVA), apresentaram diferenças significativas entre os mesmos, com níveis de significância inferiores a 0,05. Assim, percebeu-se um indicativo de que se chegara a um conjunto relativamente estável de clusters para o presente trabalho. Importante ressaltar que dos itens do instrumento final de pesquisa, a questão 1 (indicação de palavras relacionadas à felicidade) e a questão 20 (objetivo profissional) não foram incluídas na AC.

Foram processadas três análises de variância (ANOVA), sendo a última demostrada na tabela 1. 0 primeiro teste informou que os itens 12 (sig 0,120), 13 (sig 0,988), 2 (sig 0,358), 4 (sig 0,080), 7 (sig 0,074), 8 (sig 0,112), 11 (sig 0,115) não possuem níveis de significância inferiores à 0,05. O segundo informou como não significantes os itens: 6 (sig 0,062), 5 (sig 0,052 e 10 (0,063). Com a exclusão desses itens, a formação dos clusters foi efetuada com base nos itens 14 (sig 0,000), $18(0,000), 19(0,000), 3(0,045)$ e $9(0,020)$, como consta na tabela 1. Com a definição das variáveis e do agrupamento final informado na tabela 2, têm-se que cluster 2 (169) possui um maior volume de casos, sendo 5 vezes maior do que o cluster 1 (32). 
Tabela 1: Composição final dos itens significativos para a formação dos Clusters.

\begin{tabular}{|c|c|c|c|c|c|c|}
\hline \multicolumn{7}{|l|}{ ANOVA } \\
\hline & \multicolumn{2}{|l|}{ Cluster } & \multicolumn{2}{|l|}{ Erro } & \multirow[b]{2}{*}{ z } & \multirow[b]{2}{*}{ Sig. } \\
\hline & Quadrado Médio & $d f$ & $\begin{array}{l}\text { Quadrado } \\
\text { Médio }\end{array}$ & df & & \\
\hline 14. Idade & 2136,393 & 1 & 5,578 & 199 & 383,009 &, 000 \\
\hline 18. Qual a sua participação na vida econômica da família? & 26,341 & 1 &, 584 & 199 & 45,132 &, 000 \\
\hline 19. Semestre que está cursando? & 125,374 & 1 & 5,180 & 199 & 24,202 &, 000 \\
\hline 3. As minhas condições de vida são excelentes & 8,507 & 1 & 2,092 & 199 & 4,066 & 045 \\
\hline $\begin{array}{l}\text { 9. Algumas pessoas são geralmente muito felizes. Elas aproveitam a vida, } \\
\text { aconteça o que acontecer, procurando obter o máximo. Em que grau essa } \\
\text { afirmativa se aplica a você? }\end{array}$ & 9,873 & 1 & 1,796 & 199 & 5,496 &, 020 \\
\hline
\end{tabular}

Tabela 2: Quantidade de clusters e número de casos em cada grupo.

\begin{tabular}{|c|c|c|}
\hline \multicolumn{3}{|c|}{ Número de casos em cada cluster } \\
\hline Cluster & 1 & 32,000 \\
\cline { 2 - 3 } & 2 & 169,000 \\
\hline Válido & & 201,000 \\
\hline Ausente &, 000 \\
\hline
\end{tabular}

A tabela 3 relaciona de forma cruzada os casos com os clusters formados. Sua leitura permite melhor conhecer os agrupamentos e, com isso, definir seus perfis. Evidenciou-se, primeiramente, no que tange ao perfil Idade, que o cluster 1 apresentou idades que compreenderam a um intervalo entre 25 a 41 anos. E, o cluster 2 abrangeu uma faixa etária de 17 a 25 anos. Nota-se claramente a jovialidade compatível ao fator idade no cluster 2, em oposição a maturidade dos discentes do cluster 1.

Na sequência, o cluster 1 apresentou maioria dos discente nos últimos períodos do curso, o que confirma um perfil mais maduro do grupo. Por sua vez, no cluster 2 há maior volume de discentes nos primeiros semestres, corroborando a jovialidade desse grupo. No que se refere à participação na vida econômica da família, para cada discente que não trabalha no cluster 1, há 3,5 (25/7) que trabalham, seja com complemento ou não da família. No cluster 2 essa relação é inversa, para cada discente que trabalha, há 2,07 (114/55) discentes que não trabalham. Assim, no cluster 2 há predomínio de jovens, iniciantes no curso superior e que não trabalham.

Em relação às escalas adotadas pela presente pesquisa, o item 3 corresponde à escala de satisfação com a vida, sendo o único que apresentou significância na formação dos grupos. O comportamento dessa variável em relação aos clusters indica que no cluster 1 para cada respondente não satisfeitos com a vida, há 1,3 (16/12) satisfeitos, considerando as respostas acima e abaixo de 4, sendo esta indicada por 'não concordo, nem discordo'. Na mesma linha, o cluster 2 também apresenta maior proporção de satisfeitos com a vida, sendo para cada 1 insatisfeito há 3,07(117/38) satisfeitos. De todo modo, pela proporção observada, no cluster 2 há maior tendência de respostas voltadas para a satisfação com a vida, em comparação com o cluster 1 .

O item 9 corresponde à escala de felicidade subjetiva, sendo, também, o único item com significância estatística pela análise de variância (ANOVA). O cluster 1 possui uma proporção de 1 discente não feliz em relação à vida em comparação com outras pessoas, para cada 7 (28/4) felizes. O cluster 2 também informa proporção maior de felizes 3,4 (119/35), para cada 1 não feliz. De forma distinta da questão 3, observa-se 
que o grupo 1 responde por maior percentual de discentes felizes, quando comparados com à felicidades de outros.

Tabela 3: Cruzamento dos variáveis significativas por cluster formado.

\begin{tabular}{|c|c|c|c|c|}
\hline & & $\begin{array}{l}\text { Número de caso } \\
\text { de cluster }\end{array}$ & & Total \\
\hline & & 1 & 2 & \\
\hline \multirow[t]{20}{*}{ 14. Idade } & 17 & 0 & 5 & 5 \\
\hline & 18 & 0 & 21 & 21 \\
\hline & 19 & 0 & 26 & 26 \\
\hline & 20 & 0 & 36 & 36 \\
\hline & 21 & 0 & 28 & 28 \\
\hline & 22 & 0 & 25 & 25 \\
\hline & 23 & 0 & 19 & 19 \\
\hline & 24 & 0 & 8 & 8 \\
\hline & 25 & 3 & 1 & 4 \\
\hline & 26 & 7 & 0 & 7 \\
\hline & 27 & 5 & 0 & 5 \\
\hline & 28 & 3 & 0 & 3 \\
\hline & 29 & 2 & 0 & 2 \\
\hline & 30 & 3 & 0 & 3 \\
\hline & 32 & 1 & 0 & 1 \\
\hline & 33 & 2 & 0 & 2 \\
\hline & 35 & 2 & 0 & 2 \\
\hline & 36 & 2 & 0 & 2 \\
\hline & 37 & 1 & 0 & 1 \\
\hline & 41 & 1 & 0 & 1 \\
\hline Total & & 32 & 169 & 201 \\
\hline \multirow[t]{8}{*}{ 19. Semestre que está cursando? } & 1,0 & 0 & 19 & 19 \\
\hline & 2,0 & 2 & 38 & 40 \\
\hline & 3,0 & 0 & 18 & 18 \\
\hline & 4,0 & 2 & 28 & 30 \\
\hline & 5,0 & 8 & 21 & 29 \\
\hline & 6,0 & 4 & 9 & 13 \\
\hline & 7,0 & 2 & 6 & 8 \\
\hline & 8,0 & 14 & 30 & 44 \\
\hline \multirow[t]{2}{*}{ Total } & & 32 & 169 & 201 \\
\hline & & 1 & 2 & Total \\
\hline \multirow[t]{3}{*}{ 18. Qual a sua participação na vida econômica da família? } & 1,0 & 7 & 114 & 121 \\
\hline & 2,0 & 3 & 29 & 32 \\
\hline & 3,0 & 22 & 26 & 48 \\
\hline Total & & 32 & 169 & 201 \\
\hline \multirow[t]{7}{*}{ 3. As minhas condições de vida são excelentes } & 1,0 & 0 & 2 & 2 \\
\hline & 2,0 & 4 & 14 & 18 \\
\hline & 3,0 & 8 & 22 & 30 \\
\hline & 4,0 & 4 & 14 & 18 \\
\hline & 5,0 & 10 & 62 & 72 \\
\hline & 6,0 & 5 & 39 & 44 \\
\hline & 7,0 & 1 & 16 & 17 \\
\hline Total & & 32 & 169 & 201 \\
\hline \multirow{7}{*}{$\begin{array}{l}\text { 9. Algumas pessoas são geralmente muito felizes. Elas aproveitam a vida, aconteça o que } \\
\text { acontecer, procurando obter o máximo. Em que grau essa afirmativa se aplica a você? }\end{array}$} & 1,0 & 0 & 4 & 4 \\
\hline & 2,0 & 0 & 8 & 8 \\
\hline & 3,0 & 4 & 23 & 27 \\
\hline & 4,0 & 0 & 15 & 15 \\
\hline & 5,0 & 12 & 68 & 80 \\
\hline & 6,0 & 12 & 40 & 52 \\
\hline & 7,0 & 4 & 11 & 15 \\
\hline Total & & 32 & 169 & 201 \\
\hline
\end{tabular}

Em síntese, no cluster 1, predomina discentes mais maduros, embora em menor número, mais distribuídos nos semestres finais do curso e que, em geral, trabalham. Nesse grupo a satisfação com a vida, se comparada com o grupo 2 é menor, porém há maior percepção de felicidade quando os discentes se 
compararam com outros sujeitos, em relação ao grupo 2. No cluster 2 prepondera jovens que estão iniciando o curso superior, que, em geral, não trabalham. São mais satisfeitos com a vida, embora menos felizes do que o grupo 1. Assim, considera-se importante destacar que a concepção sobre satisfação com a vida e sobre a felicidade subjetiva são apreciadas de forma distinta pelos grupos, mesmo diante de uma forte correlação entre as duas questões, como observado na tabela 4.

Ainda em relação aos itens da escala de satisfação com a vida, no caso, as minhas condições de vida são excelentes, teve, no cluster 1, uma média de 4,2. Com este resultado interpreta-se que o grupo 1 é formado por pessoas que consideram a vida pouco excelentes, ou talvez, são um pouco satisfeitas em relação às suas condições . No segundo grupo, a média foi de 4,78 , o que corrobora a definição do perfil desse cluster como mais satisfeitos do que o grupo 1 , haja vista que o valor é superior à média do grupo 1 . Assim, em média, os dois grupos são poucos satisfeitos, mais o segundo grupo indica maior satisfação em comparação com o primeiro.

No que diz respeito à escala de felicidade subjetiva, especificamente a questão obtida pela análise de variância, sendo a avaliação de felicidade mensurada com base no aproveitar a vida ao máximo, em comparação com outros, no cluster 1 a média de respostas foi de 5 e, no cluster 2 de 4,76. Depreende-se, portanto, que o primeiro grupo apresenta, em geral, maior percepção de felicidade subjetiva em comparação com o grupo 2, embora, da mesma forma, as médias se aproximam da pouca aplicabilidade do item ao grupo (aplica-se um pouco em mim), o que denota pequena percepção de felicidade pelos grupos. Com base nos resultados demostrados na figura 1 e tabelas 1, 2, 3 e 4, a discussão aprecia a formação dos clusters e, especificamente, a relação do perfil descrito com a satisfação com a vida e com a felicidade subjetiva, compreendidas nesse trabalho como requisitos da percepção de felicidade.

Tabela 4: Correlação entre os itens de satisfação com a vida e felicidade subjetiva.

\begin{tabular}{|c|c|c|c|c|}
\hline & & & \begin{tabular}{|c|} 
3. As minhas \\
condições de vida \\
são excelentes
\end{tabular} & $\begin{array}{c}\text { 8. Comparativamente com as outras } \\
\text { pessoas como eu, considero-me } \\
\text { uma pessoa totalmente feliz }\end{array}$ \\
\hline \multirow{6}{*}{$\begin{array}{l}\text { Spearman's } \\
\text { rho }\end{array}$} & \multirow{3}{*}{$\begin{array}{l}\text { 3. As minhas condições de vida são } \\
\text { excelentes }\end{array}$} & $\begin{array}{l}\text { Correlation } \\
\text { Coefficient }\end{array}$ & 1,000 &, $884^{* *}$ \\
\hline & & Sig. (1-tailed) & &, 000 \\
\hline & & $\mathrm{N}$ & 201 & 201 \\
\hline & \multirow{3}{*}{$\begin{array}{l}\text { 8. Comparativamente com as outras } \\
\text { pessoas como eu, considero-me } \\
\text { uma pessoa totalmente feliz }\end{array}$} & $\begin{array}{l}\text { Correlation } \\
\text { Coefficient }\end{array}$ &, $884^{* *}$ & 1,000 \\
\hline & & Sig. (1-tailed) & ,000 & \\
\hline & & $\mathrm{N}$ & 201 & 201 \\
\hline
\end{tabular}

\section{DISCUSSÃO}

As três palavras mais citadas como referentes à felicidade (Família, Amor e Dinheiro) ressalta o caráter genérico da felicidade como organizador da vida humana (CARVALHO, 2010), posto que as três dimensões respondem pela vida familiar, pelas interações afetuosas desenvolvidas no campo social e pela necessidade de sobrevivência, por vezes, estendido à aquisição de bens e serviços que proporcionam maior conforto e bem-estar, conquistado pelo dinheiro. Sendo este o terceiro elemento considerado, e, pela estrita 
relação do dinheiro com o trabalho, percebe-se a relevância do trabalho como constitutivo de felicidade, o que situa as discussões de Paschoal et al. (2010) e Carvalho et al. (2010).

Com base nos agrupamentos e no cruzamento das variáveis por cluster foi possível identificar dois perfis. De forma geral, a definição prévia dos grupos buscou caracterizá-los em função de dois grupos com menor e maior percepção de felicidade subjetiva e de satisfação com a vida. De início, pelo teste de variância, foi excluída a questão sobre sexo, considerando a não significância dessa categoria para a formação dos grupos. Assim, os dados da pesquisa indicam que não há distinção entre maior e menor satisfação e felicidade em função do sexo, considerando que o sexo estaria presente de forma homogênea nos dois grupos. Tal fato, caminha para a não distinção de felicidade por homens e mulheres, o que é perceptível nas pesquisas de Wilson (1967) e Guedea et al. (2006).

Em seguida, com a exclusão de outras variáveis, foram confirmadas a idade, o semestre e a participação econômica nos grupos. Nesse caso, é possível considerar esperado que em um grupo com maior percentual de jovens, seja também aquele que possui maior volume de discentes nos primeiros semestres do curso e que, também, não trabalha. Assim, seu oposto informa a formação do segundo grupo. $O$ que de fato colabora com uma maior discussão sobre o tema felicidade com suporte na satisfação com a vida e com a percepção de felicidade subjetiva está na análise de como essas categorias foram consideradas pelos grupos.

Sendo a satisfação com a vida representada pelo item que informa as condições gerais de vida, construto que responde pela vida de forma geral, sem distinção de domínios específicos (DIENER et al., 1985; PAVOT et al., 1993). No que tange à felicidade subjetiva há referência a uma avaliação pessoal, que envolve cognições e afetos, sobre o sentido e o significado da felicidade (LYUBOMIRSKY et al., 1999). Nesse caso, o bem-estar relativo é inquirido pela questão 9, de forma a situar o bem-estar em função de outros (SELIGMAN et al., 2000; RODRIGUES et al., 2010).

Em relação aos dois constructos, o grupo 1 (mais jovens) informa que os discentes são mais satisfeitos com a vida, embora menos felizes se comparados ao grupo 2 (mais maduros). Nesse caso, percebe-se que diante da questão que envolve maior avaliação sobre o bem-estar, em que duas situações devem ser elaboradas: como eu me sinto e, como base nisso, como estou em comparação ao outro, os jovens se posicionam menos felizes, embora satisfeitos. Ao mesmo tempo, sendo o grupo 2 menos satisfeitos, porém mais felizes, processam uma avaliação pessoal mais propensa à felicidade.

\section{CONSIDERAÇÕES FINAIS}

Pesquisas sobre felicidade poderiam ser mais amplamente exploradas, considerando o impacto que a felicidade desempenha na vida dos sujeitos. Nesse sentido, a pesquisa empreendeu análise sobre clusters na busca de descrever grupos que percebem felicidade de formas distintas. Essa tarefa situa a problemática de compreender características de sujeitos que se percebem mais felizes e, com base nisso, refletir, no futuro, sobre estratégias de ação. 
Diante da formação dos clusters e da caracterização dos grupos observa-se coerência em termos de que os mais jovens são aqueles que, em geral, não trabalham e também são iniciantes no curso superior. Ao mesmo, os mais maduros representam os discentes que trabalham e estão na fase final do curso. No entanto, o grupo 1, dos mais jovens, é formado por sujeitos mais satisfeitos com a vida, embora menos felizes, se comparados com o grupo 2. Este, por sua vez, com respondentes mais maduros, representa agrupamento de discentes menos satisfeitos, porém mais felizes se comparados ao grupo 1.

Mesmo diante da análise por cluster e, com base nas médias dos itens 3 e 9, ressalta-se que os discentes indicam pouca percepção de felicidade, haja vista que as respostas giram em torno da pouca concordância sobre possuírem condições de vida excelentes (item 3) e pouca aplicabilidade da afirmativa sobre o aproveitamento da vida ao máximo (item 9).

No que que tange à avaliação subjetiva de felicidade, indaga-se, porém, se o fato de serem mais maduros os sujeitos possuiriam maior capacidade de avaliação subjetiva de felicidade, especificamente se considerar melhor leitura de contexto e percepção das situações que estão às suas voltas, advindos de uma maior experiência de vida. Por outro lado, sendo os jovens mais imediatistas, seriam mais propensos a fazer uma análise mais generalista sobre satisfação, possuindo, então, maior dificuldade em distinguir o que significa felicidade subjetiva própria, assim como análise da felicidade daqueles que estão a sua volta. Essas questões precisariam ser melhor exploradas para que se possa gerar inferências mais atentes a relação suposta (idade/melhor avaliação da felicidade), objeto de análise para pesquisas futuras.

De todo modo, além de a própria pesquisa permitir reflexão sobre o tema e possível abertura à discussão a respeito, acredita-se que novas pesquisas também podem favorecer a construção de agendas de diálogos e de ações sobre felicidade, tanto no âmbito da academia, campo estudado, como no contexto laboral, em função da pretensa qualidade de vida no trabalho.

Por fim, o estudo limitou-se a apresentação dos dados pela caracterização dos grupos formados. A técnica escolhida revelou potencial para esta tarefa, mas o volume de itens excluídos pela análise de variância (ANOVA) limitou essa caracterização a poucas variáveis. Tal fato pode ser reflexo de sobreposição de questões (itens que medem a mesma coisa) o que pode ser minimizado pela utilização de escala única com itens dimensionados e validados por análises confirmatórias (Análise Fatorial Confirmatória, por exemplo).

Assim, como sujeitos capazes portadores de felicidade, discentes podem ser inquiridos acerca da sua felicidade, bem como sobre o que concebem como felicidade, com apreciação que contemple valores pessoais, organizacionais e culturais. Para tanto, seria necessário pensar, também, em agendas de pesquisas capazes de aprofundar a própria concepção de felicidade.

\section{REFERÊNCIAS}

ALBUQUERQUE, A.; TRÓCCOLI, B. T.. Desenvolvimento de uma escala de bem-estar subjetivo. Psicologia, Teoria e

Pesquisa, v.20, n.2, p.153- 164, 2004.

AMABILE, T. M.. Affect and creativity at work.

Administrative Science Quaterly, v.50, n.3, p.367-403, 2005.
BENDASSOLI, P. F.; MALVEZZI, S.. Desempenho no Trabalho: definições, modelos teóricos e desafios à gestão. In: BORGES, L. O.; MOURÃO, L.. O Trabalho e as Organizações: atuações a partir da Psicologia. São Paulo: Artmed, 2013.

CANTRIL, H.. The pattern of human concerns. New Brunswick: Rutgers University Press, 1965. 
CARVALHO, M. B.. A felicidade na agenda da administração e suas relações com conceitos organizacionais. Dissertação (Mestrado) - Universidade Fumec, Belo Horizonte, 2010.

CARVALHO, M. B.; GONÇALVES, C. A.; PARDINI, D. J.. A felicidade em foco: mensurando conceito metafísico para estratégia governamental e recomendações organizacionais. Revista de Administração da UFSM, Santa Catarina, v.3, p.269-187, 2010.

CLONINGER, C. R.. Feeling good: the science of well-being. New York: Oxford University Press, 2004.

COLETA, J. A.; COLETA, M. F.. Felicidade e bem-estar subjetivo entre professores universitários. Quaestio, v.9, n.2, p.131-143, 2007.

COLETA, J. A.; COLETA, M. F.. Felicidade, bem-estar subjetivo e comportamento acadêmico de estudantes universitários. Psicologia em Estudo, v.11 n.3, p.533-539, 2006.

COLETA, J. A.; LOPES, J. E. F.; COLETA, M. F.. Felicidade, bemestar subjetivo e variáveis sócio demográficas, em grupos de estudantes universitários, Psico-USF, v.17, n.1, p.129-139, 2012.

COLLIS, J.; HUSSEY, R.. Pesquisa em administração: um guia prático para alunos de graduação e pós graduação. São Paulo: Bookman, 2006.

CORBI, R. B.; MENEZES FILHO, N. A.. Os determinantes empíricos da felicidade no Brasil. Revista de Economia Política, v.26, n.4, p.518-536, 2006.

COSTA, L. S. M.; PEREIRA, C. A. A.. Bem-estar subjetivo: aspectos conceituais. Arquivos Brasileiros de Psicologia, v.59, n.1, p.72-80, 2007.

DEMO, D. H.; ACOCK, A. C.. Singlehood, marriage, and remarriage: the effects of family structure and family relationships on mother's well-being. Journal of Family Issues, v.17, p.388-407, 1996.

DIENER, E.. Subjective well being. Psychological Bulletin, v.95, n.3, p.542-575, 1984.

DIENER, E.. The Science of well-being: the collected works of Ed Diener. New York: Springer, 2009.

DIENER, E.; EMMONS, R. A.; LARSEN, R. J.; GRIFFIN, S.. The Satisfaction with Life Scale. Journal of Personality

Assessment, v.49, n.1, p.71-75. 1985.

DIENER, E.; OISHI, S.. Money and happiness: income and subjective well-being across nations. In: DIENER, E.; SUH, E. M.. Culture and subjective well-being. Cambridge: The MIT Press, 2000. p.185-218.

DIENER, E.; SUH, E. M.. Culture and subjective well-being. Cambridge: The MIT Press, 2000.

DIENER, E.; SUH, E. M.; LUCAS, R. E.; SMITH, H. L.. Subjective well-being: three decades of progress. Psychological Bulletin, v.125, n.2, p.276-302, 1999.

FERRAZ, R. B.; TAVARES, H.; ZILBERMAN, M. L.. Felicidade: uma revisão. Revista de Psicologia Clínica, v.34, n.5, p.234242, 2007.
FOWLER, J. H.; CHRISTAKIS, N. A.. The dynamic spread of happiness in a large social network: longitudinal analysis over 20 years in the framingham heart study. British Medical Journal, v.337, p.2338, 2008.

GIACOMONI, C. H.. Bem-estar subjetivo: em busca de qualidade de vida. Temas em Psicologia da SBP, v.12, n.1, p.43-50, 2004.

GONÇALVES, D. M.; KAPCZINSKI, F.. Transtorno mental, indicadores demográficos e satisfação com a vida. Revista de Saúde Pública, v.42, n.6, p.1060-1066, 2008.

GUEDEA, M. T. D.; ALBUQUERQUE, F. J. B.; TRÓCCOLI, B. T.; NORIEGA, J. A. V.; SEABRA, M. A. B.; GUEDEA, R. L. D.. Relação do bem-estar subjetivo, estratégias de enfrentamento e apoio social em idosos. Psicologia Reflexão e Crítica, v.19, n.2, p.301-308, 2006.

HAIR JUNIOR, J. F.. Análise Multivariada de Dados. 6 ed. Porto Alegre: Bookman, 2009.

HUME, D.. Ensaios morais, políticos e literários. 2 ed. São Paulo: Abril Cultural, 1980.

INGLEHART, R.. Culture shift in advanced industrialized society. Princeton: Princeton University Press, 1990.

INGLEMAN, R.; KLINGEMANN, H. D.. Genes, culture, democracy and happiness. In: DIENER, E.; SUH, E. M. Culture and subjective well-being. Cambridge: The MIT Press, 2000. p.165-167.

KAHNEMAN, D.; KRUEGER, A. B.; SCHKADE, D.; SCHWARZ, N.; STONE, A. A.. Would you be happier if you were richer? A foccusing illusion. Science, v.30, n.312, p.1908-1910, 2006.

LUCAS, R. E.; GOHM, C. L.. Age and sex differences in subjective well-being across cultures. In: DIENER, E.; SUH, E. M.. Culture and subjective well-being. Cambridge: The MIT Press, 2000. p.291-317.

LYKKEN, D.; TELLEGEN, A.. Happiness is a stochastic phenomenon. Psychological Science, v.7, p.186-189, 1996.

LYUBOMIRSKY, S.. Why are some people happier than others?. American Psychologist, v.56, p.239-249, 2001.

LYUBOMIRSKY, S.; LEPPER, H.. A measure of subjective happiness: preliminary reliability and construct validation. Social Indicators Research, v.46, p.137-155, 1999.

LYUBOMIRSKY, S.; SHELDON, K. M.; SCHKADE, D.. Pursuing happiness: the architecture of sustainable change. Review of General Psychology, v.9, p.111-131, 2005.

MCMAHON, D.. Happiness: a history. New York: Atlantic Monthly Press, 2006.

OLIVEIRA, G. F.; BARBOSA, G. A.; SOUZA, L. E. C.; COSTA, C. L., ARAÚJO, R. C. R.; GOUVEIA, V. V.. Satisfação com a vida entre profissionais de saúde: correlatos demográficos e laborais. Revista Bioética, v.17, n.2, p.319-334, 2009.

OSWALD, J. A.; PROTO, E.; SGROI, D.. Happiness and Productivity. Conventry, University of Warwick, 2009. 
OTTA, E.; FIQUER, J. T.. Bem-estar subjetivo e regulação de emoções. Psicologia em Revista, v.10, n.15, p.144-149, 2004.

PASCHOAL, T.; TORRES, C. V.; PORTO, J. B.. Felicidade no trabalho: relações com suporte organizacional e suporte social. Revista Administração Contemporânea, Curitiba, v.14, n.6, p.1054-1072, 2010.

PASSARELI, P. M.; SILVA, J. A.. Psicologia positiva e o estudo do bem-estar subjetivo. Estudos de Psicologia, v.24, n.4, p.513-517, 2007.

PAVOT, W.; DIENER, E.. Review of the satisfaction with life scale. Psychological Assessment, v.5, n.2, p.164-172, 1993.

POHLMANN, M. C.. Análise de conglomerados. In: CORRAR, L. J.; PAULO, E.; DIAS FILHO, J. M.. Análise Multivariada: para os cursos de Administração, Ciências Contábeis e Economia. São Paulo: Atlas, 2009.

RIBEIRO, J. V.. Em busca da felicidade: momentos, visões e impacto: estudo de uma família. Dissertação (Mestrado Integrado em Psicologia) - Universidade de Lisboa, Lisboa, 2009.

RICHARDSON, R. J.. Pesquisa social: métodos e técnicas. 3 ed. São Paulo: Atlas, 1999.

RODRIGUES, A.; SILVA, J. A.. O papel das características sócio demográficas na felicidade. Psico-USF, v.15, n.1, 113-123, 2010.

RODRIGUES, S. B.. Desafios da administração no século XXI. Revista de Administração de Empresas, São Paulo, v.44, p.97-107, 2004.

SALOVEY, P.; ROTHMAN, A. J.; STEWARD, W. T.. Emotional states and physical health. American Psychologist, v.55, n.1, p.110-121, 2000.

SCALCO, D. L.. Auto percepção de felicidade e fatores associados em adultos de uma cidade do sul do Brasil: estudo de base populacional. Dissertação (Mestrado em
Epidemiologia) - Universidade Federal de Pelotas, Pelotas, 2008.

SELIGMAN, M. E. P.; CSIKSZENTMIHALYI, M.. Positive psychology: an introduction. American Psychology, v.55, p.5-14, 2000.

SELIGMAN, M.. Felicidade autêntica. Rio de Janeiro: Objetiva, 2004.

STOCK, W. A.; OKUN, M. A.; HARING, M. J.; WITTER, R. A.. Age and subjective well-being: a meta-analysis. In: LIGHT, R. J.. Evaluation studies: review annual. 8 ed. Beverly Hills: Sage, 1983. p.279-302.

VEENHOVEN, R.. Measures of gross national happiness. In: OECD WORLD ECONOMIC FORUM ON STATISTICS: 'ROUNDTABLE ON “MEASURING, KNOWLEDGE AND POLICY'. Anais. Istanbul: OECD, 2007.

VEENHOVEN, R.. Progres dans la comprehension du bonheur. Revue Québécoise de Psychologie, v.18, p.29-74, 1997.

VEENHOVEN, R.. Questions on happiness: classical topics, modern answers, blind spots. In: STRACK, F.; ARGYLE, M.; SCHWARTZ, N.. Subjective well-being: an interdisciplinary perspective. New York: Pergamon, 1991. p.7-26.

VERGARA, S. C.. Projetos e relatórios de pesquisa em administração. 11 ed. São Paulo: Atlas, 2009.

VICK, T. E.. Desafios organizacionais do século XXI: Uma introdução ao fórum sobre inovação, capacidades dinâmicas, capacidade de absorção do conhecimento e relações simbólicas. Revista de Administração Mackenzie, São Paulo, v.16, n.3, p.14-19, 2015.

WATSON, D.. Mood and temperament. New York: The Guilford Press, 2000.

WILSON, W.. Correlates of avowed happiness. Psychological Bulletin, v.67, p.294-306, 1967. 